\title{
MOVPE issues in the development of ordered GaInP metamorphic buffers for multijunction solar cells
}

Manuel Hinojosa, Iván García and Carlos Algora
Oscar Martínez

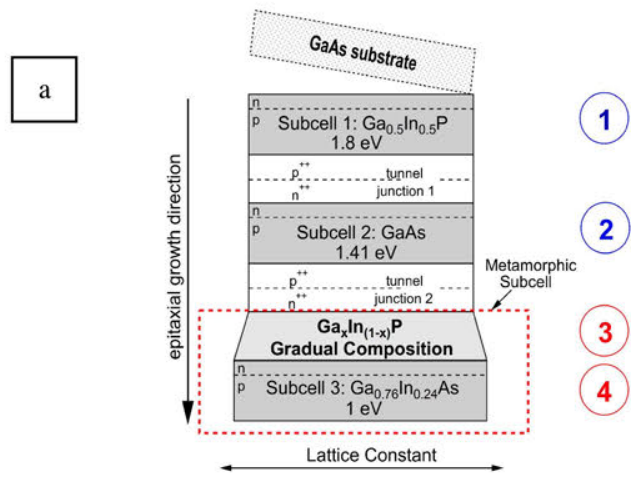

b

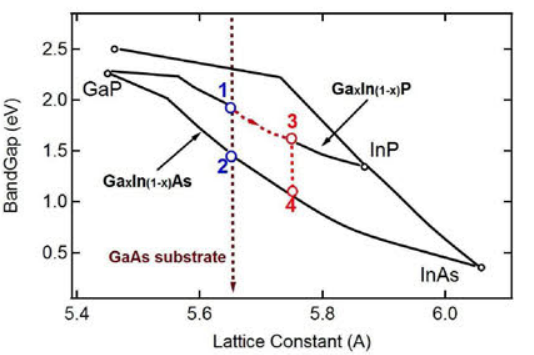

Fig. 1. (a) Structure of the 3-junction inverted metamorphic solar cell developed at IES-UPM and (b) lattice constant route followed during the metamorphic III-V alloys growth.

This work aims to discuss several growth technical issues arisen during the implementation of a GaInP CGB in our MOVPE reactor. These considerations are focused on achieving high quality ordered-GaInP metamorphic buffers by increasing $\mathrm{PH} 3$ partial pressure and growth rates (GR), and attaining precise control of the GaInP compositions under different growth conditions. The last section is devoted to presenting the metamorphic $1 \mathrm{eV}$ GaInAs subcell developed using these $\mathrm{CGBs}$ and the inverted metamorphic 3-junction solar cell implemented.

\section{EXPERIMENTAL METHOD}

Metamorphic buffer samples with the structure shown in Fig. 2 were grown in a commercial horizontal MOVPE lowpressure reactor (Aixtron AIX200/4) equipped with an in-situ reflectance (R) and reflectance anisotropy (RA) spectrometer

This work has been supported by the Spanish MINECO through the project TEC2014-54260-C3-1-P and by the Comunidad de Madrid through the project MADRID-PV (S2013/MAE-2780). M. Hinojosa is funded by the Spanish MINECD through the FPU15 grant. I. García is funded by the Spanish Programa Estatal de Promoción del Talento y su Empleabilidad through a Ramón y Cajal grant.

\begin{abstract}
Metamorphic solar cells require high-quality compositional graded buffer (CGB) layers to perform as virtual
substrate. Several technical growth issues arisen during the implementation of an ordered-GaInP CGB at IES-UPM MOVPE on achieving high-quality metamorphic buffers by increasing phosphine partial pressure and growth rates, which requires different GaInP alloys. Improvements on the material quality are proved. It is also presented a $1 \mathrm{eV}$ GaInAs metamorphic subcell
\end{abstract}

Metamorphic epitaxy provides access to a wide palette of
-V semiconductor material compositions (bandgaps) lattice efficiencies over $43 \%$ [4] and provides a basis for a fourth 0.7 eV junction by the introduction of an additional CGB. This configuration contains a $1 \mathrm{eV}$ GaInAs metamorphic subcell $2 \%$ mismatched to GaAs that is covered by a GaInP buffer layer. 
(LayTec EpiRAS 200/4) with true temperature measurement capability by emissivity-corrected pyrometry. The precursors used were $\mathrm{AsH}_{3}$ and $\mathrm{PH}_{3}$ for group-V and TMGa and TMIn for group-III elements.
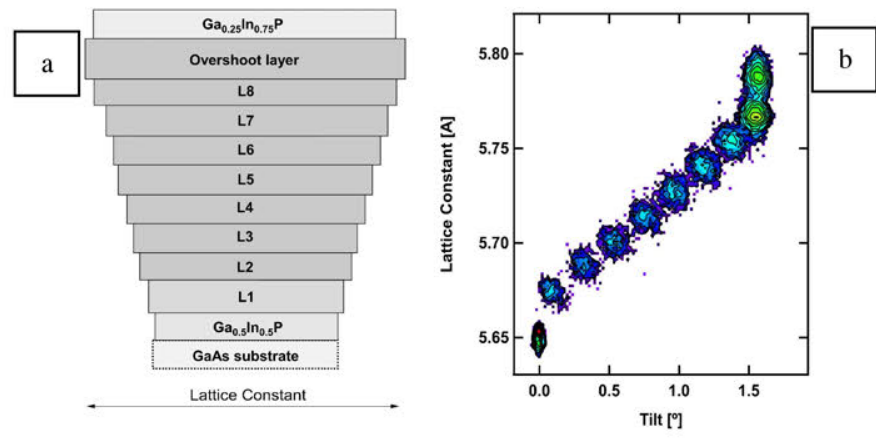

Fig. 2. (a) Structure of the GaInP CGB design that covers the lattice mismatch up to $1 \mathrm{eV}$ GaInAs subcell. It includes eight $250 \mathrm{~nm}$ and overshoot layers each one $0.25 \%$ lattice-mismatched to the previous one. (b) Reciprocal Space Map of a CGB sample.

Structural material properties and composition of GaInP layers have been measured by ex-situ high resolution X-Ray diffraction (XRD) with an X-Pert Panalytical diffractometer. Cathodoluminescence (CL) was used to spatially resolve threading dislocations and estimate the threading dislocation density (TDD) using a XICLone (Gatan UK) CL system attached to a LEO 1530 (Carl-Zeiss) FESEM available at the GdS-Optronlab Group (Universidad de Valladolid). Surface roughness was characterized by Atomic Force Microscope (AFM) with a Digital Instruments-Multimode IIIa microscope available at Centro Nacional de Microscopía Electrónica.

\section{DEVELOPMENT OF METAMORPHIC BUFFERS}

Elastic strain accumulated through the mismatch growth of thick layers is released by the introduction of misfit dislocations, which lie parallel to the mismatch interface, and threading dislocations, which go through the semiconductor bulk up to the surface and act as recombination centers for photogenerated carriers. An example of these dislocations is shown in the CL image in Fig. 3. The strain introduced by the growth of the subsequent mismatched layers acts as drive force for dislocation gliding, resulting in a higher average length of the misfit segments and a reduction of the threading dislocation density for a given mismatch grade [5]. The goal of the CGB is to confine the unavoidable misfit segments in a buffer inactive layer and to release the mismatch strain energy by dislocation gliding instead of nucleation of new dislocations. For this purpose, it is crucial to control different growth parameters as well as each layer composition.

For instance, low surface roughness reduces nucleation of new dislocations and promotes its gliding [6]. Although crosshatch roughness, illustrated in an AFM image in Fig. 3, typically develops during metamorphic growth, it can be reduced by increasing the phosphine partial pressure and the growth rate, as demonstrated for ordered-GaInP buffers [7]. Thus, these growth parameters are explored in order to improve CGB quality, which is evaluated with TDD, RMS roughness and the Full Width at Half Maximum (FWHM) of the XRD Reciprocal Space Map layer peak.

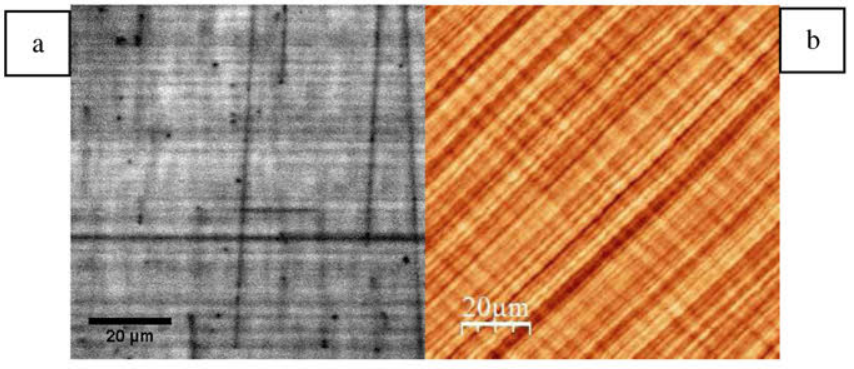

Fig. 3. (a) CL plan view image of a CGB sample. Threading dislocations corresponds to the dark spots and misfit dislocations to the dark lines.

(b) Characteristic crosshatch roughness can be seen in the AFM image.

Modification of phosphine partial pressure and GR in a low-pressure reactor is challenging given their intricate crossrelation with other growth parameters. In our case, phosphine partial pressure used at its typical highest value, 1.8 mbar, proved to be insufficient to attain high quality metamorphic buffers and required to be changed. In a low pressure-reactor, the phosphine partial pressure can be raised by increasing the reactor chamber pressure, which modifies the fluidodynamics and requires recalibration of all growth processes involved, and by increasing the phosphine flow, which have a direct effect on the total phosphine consumption in a growth process. In this way, phosphine partial pressure has been increased up to 8.9 mbar, by combining the two options, enhancing drastically the CGB quality, as shown in Fig. 4.

Increasing the growth rate has an obvious interest as reduces the growth time and, hence, the epitaxy cost. The GR, in the mass-transport-limited growth process region, depends linearly on the group-III precursors molar flows. So, TMIn molar flow had to be risen in two ways: by increasing the carrier flow injected into the bubbler and by raising the metalorganic concentration in the vapor phase. However, due to the characteristic low evaporation rate of TMIn, the concentration of metal-organic extracted from the bubbler for high carrier gas flows decreases inducing a non-lineal behavior and instability (see Fig. 5). Hence, the modification of the bubbler temperature becomes the most suitable manner to increase the GR. By changing the temperature from $17^{\circ} \mathrm{C}$ to $25^{\circ} \mathrm{C}$, TMIn concentration has been doubled (Fig. 5) allowing to double the molar flow without modifying the injected carrier flow. Therefore, with the corresponding TMGa molar flow adjustment, the GR of the lattice-matched GaInP of the CGB has been successfully risen from 3.7 to $6.7 \mu \mathrm{m} / \mathrm{h}$. Another way to mitigate the evaporation-rate limitation consists of connecting two TMIn bubblers in series. In Fig. 5 it is also shown how this configuration allows using higher carrier gas flows without seeing a change in the TMIn concentration.

Table 1 shows a comparison between two representatives CGB grown at both commented GR. It can be noted that GR increase has a positive impact on the metamorphic buffer quality. The roughness RMS value decreases from 15 to 10.7 $\mathrm{nm}$ and the crystalline quality improves as indicated by the decrease in the FWHM. The nominal composition of the GaInP virtual substrate has been more precisely achieved in the CGB with higher GR resulting in a higher mismatch grade. This is expected to be the cause of achieving a slightly higher TDD in the CGB with a higher growth rate. 


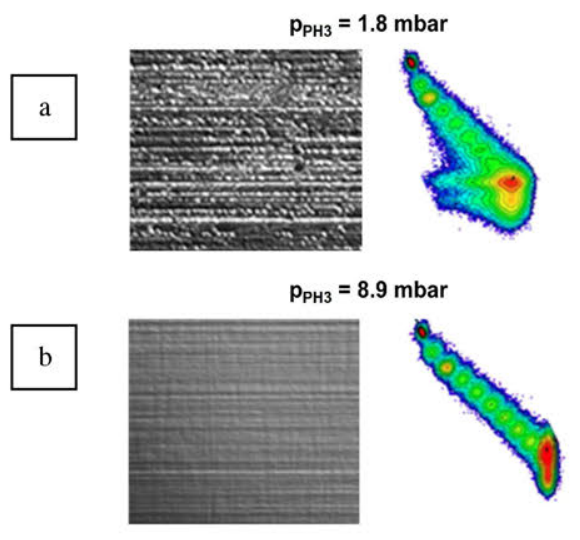

Fig. 4. Nomarski microscope pictures (left) and reciprocal space map around the 004 reflection along the [1-10] direction (perpendicular to substrate miscut steps) (right), for CGBs grown with low (a) and high (b) partial pressure of $\mathrm{PH}_{3}$.

Additionally, sample surface temperature can be modified during epitaxy due to changes on its conductivity and impedance of the chamber gases. This gives rise to significant temperature variations along a growth process, with observed absolute variations over $15^{\circ} \mathrm{C}$ between the first and the last CGB layer. This temperature drift could be affecting the incorporation of group-III elements into GaInP and, as a consequence, the composition of the grown material.

However, as can be seen in Fig. 6, the molar flows ratio introduced into the reactor chamber $\left(\mathrm{f}_{\mathrm{Ga}} / \mathrm{f}_{\mathrm{In}}\right)$ and the stoichiometric ratio obtained for the different CGB layers $\left(\mathrm{x}_{\mathrm{Ga}} / \mathrm{x}_{\mathrm{In}}\right)$ fits with a linear regression. This linearity suggests that the distribution coefficient ratio is not changing for the temperature range observed during the growth.

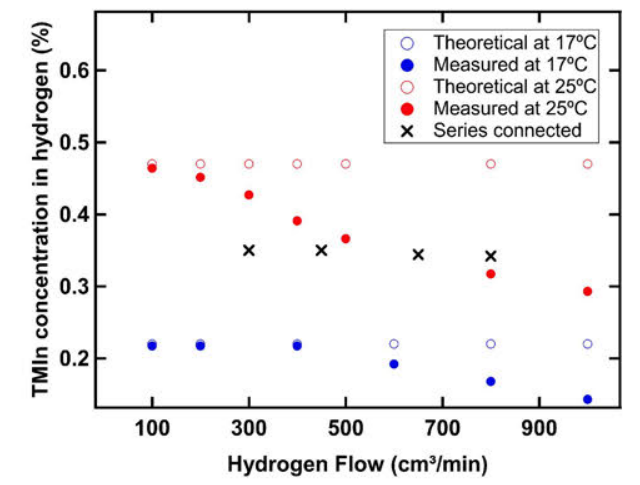

Fig. 5. Evolution of extracted TMIn concentration as a function of carrier flow for different bubbler temperatures. It exhibits sublinearity due to TMIn low evaporation rate.

TABLE I.

QUALITY COMPARISON BETWEEN TWO CGBS

\begin{tabular}{|c|c|c|}
\hline GR $(\mu \mathrm{m} / \mathrm{h})$ & 3.7 & 6.7 \\
\hline Composition (\%Ga) & 28 & 23 \\
\hline Mismatch (\%) & 1.7 & 2.2 \\
\hline Roughness RMS (nm) & 15.7 & 10.8 \\
\hline TDD $\cdot 10^{5}\left(\mathrm{~cm}^{-2}\right)$ & 6.7 & 7.2 \\
\hline Omega FWHM (arcsec) & 655 & 370 \\
\hline
\end{tabular}

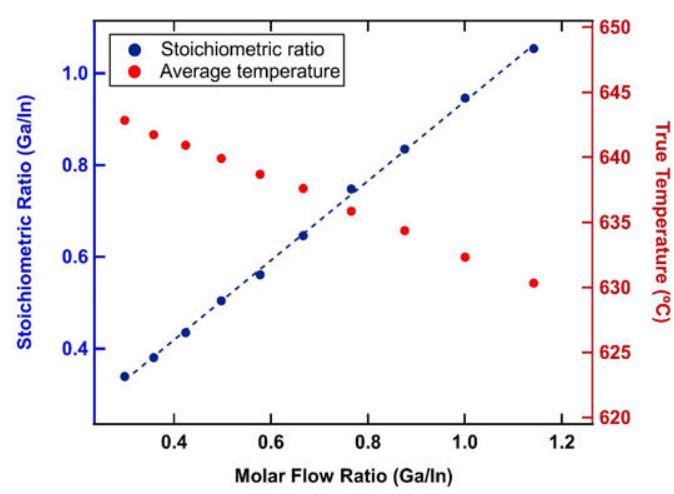

Fig. 6. Experimental relationship between the molar flow ratio introduced into the reactor chamber $\left(\mathrm{f}_{\mathrm{Ga}} / \mathrm{f}_{\mathrm{In}}\right)$ and stoichiometric $\mathrm{GaInP}$ ratio $\left(\mathrm{x}_{\mathrm{Ga}} / \mathrm{x}_{\mathrm{In}}\right)$ obtained for the different CGBs layers. The average temperature in which each layer was grown is also displayed.

\section{INVERTED METAMORPHIC TRIPLE JUNCTION SOLAR CELL}

An inverted metamorphic single junction $1 \mathrm{eV}$ GaInAs solar cell using a GaAs substrate and the ordered-GaInP CGB explained in the previous section, with a GR of $3.7 \mu / \mathrm{h}$ and a phosphine partial pressure of 8.9 mbar, has been implemented.

The External Quantum Efficiency (EQE) of the GaInAs solar cells fabricated is shown in Fig. 7. Firstly, it can be observed that the subcell bandgap is slightly higher than the 1 $\mathrm{eV}$ targeted. The reason is that the GaInP virtual substrate presents a deviation from the nominal composition being the final mismatch grade lower than $2 \%$. However, the composition of CGB layers have been tweaked for the newest GR, as explained before, resulting in a virtual substrate already lattice-matched to $1 \mathrm{eV}$ GaInAs alloy, which will be used to grow GaInAs subcells in the near future

The carrier collection of the device, which directly depends on the material quality, is good but can be clearly improved. The two growth temperatures used show virtually the same EQE, which can indicate that either it does not affect significantly to the quality of the material, or this quality is limited by other factors such as the TDD or unwanted misfit dislocations in the solar cell photoactive layers. The improvement of this subcell is an ongoing work.

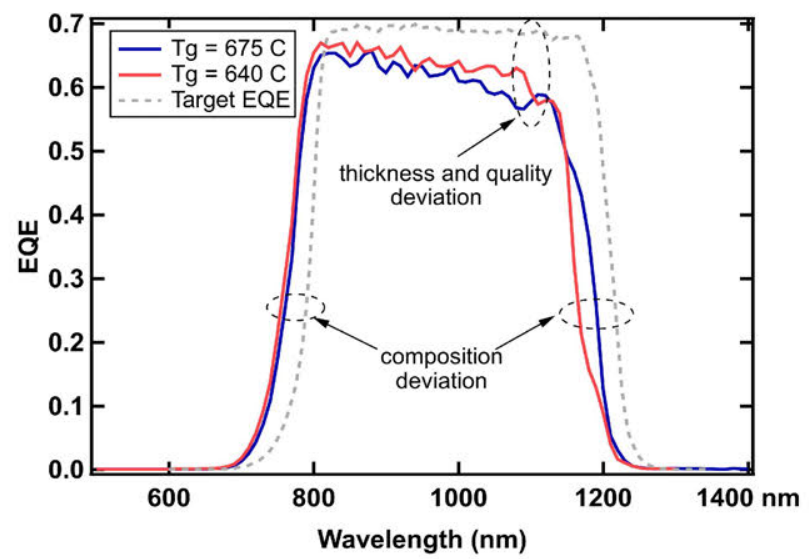

Fig.7. External Quantum Efficiency (EQE) of the metamorphic single junction $1 \mathrm{eV}$ GaInAs cells developed. The grey curve corresponds to a state-of-the-art GaInAs solar cell [8] 
Finally, the $1 \mathrm{eV}$ GaInAs subcell has been included in a 3junction inverted metamorphic solar cell (Fig. 1). The EQE and light Current-Voltage (IV) curves are presented in Fig. 8. In relation to the quality of the lattice-matched subcells it is in line with the historical GaInP and GaAs developed at IES-UPM confirming that the inverted configuration has been developed successfully. Besides, the EQE shows the same performance of the $1 \mathrm{eV}$ GaInAs subcell in the triple-junction as in the singlejunction. Moreover, the 3-junction solar cell exhibits almost the same open-circuit voltage (Voc) as the addition of the single junction ones. Taken together, these results suggest that the 3junction inverted metamorphic solar cell has been successfully implemented with a good performance of the $1 \mathrm{eV}$ GaInAs bottom cell. A parasitic junction can be observed, though, which spoils the FF of the triple-junction solar cell. Eliminating this parasitic junction, which was found to be originated in the top tunnel junction, is underway.
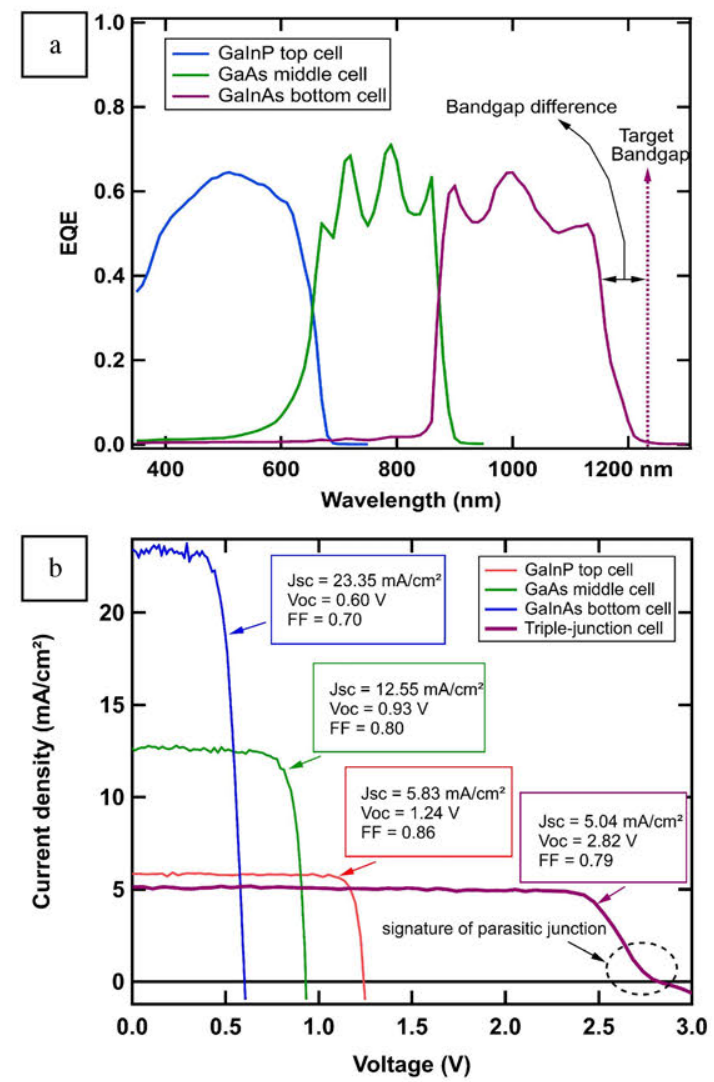

Fig.8. (a) EQE and (b) light Current-Voltage (IV) curve of the 3-junction inverted metamorphic and inverted single junction subcells implemented at IES-UPM. The IV curves are taken using the light from a solar simulator based on a Xe-lamp without spectral correction, at an intensity $\sim 1$ sun.

\section{SUMMARY}

In this work some MOVPE growth technical issues arisen during the development of ordered-GaInP CGB have been described. In this way, an improvement of the CGB which covers the $2 \%$ mismatch between GaAs and $1 \mathrm{eV}$ GaInAs have been proved by increasing the phosphine partial pressure and the growth rate. TDD, RMS roughness and FWHM in RSM peaks have been used to assess the higher quality.
Metamorphic $1 \mathrm{eV}$ GaInAs subcell using not yet optimized CGBs have been presented and discussed in CGB terms. The inverted metamorphic 3-junction solar cell, including the $1 \mathrm{eV}$ GaInAs as bottom cell, has been also presented, demonstrating the successful integration of the metamorphic subcell into an inverted multijunction configuration.

\section{ACKNOWLEDGMENT}

The authors acknowledge Jesús Bautista and Luis Cifuentes for their valuable help and the GdS-OptronLab group from University of Valladolid for allowing the use of their CL system.

\section{REFERENCES}

[1] R. M. France et al., "Design Flexibility of Ultrahigh Efficiency FourJunction Inverted Metamorphic Solar Cells,” IEEE J. Photovolt., vol. 6, no. 2, pp. 578-583, Mar. 2016.

[2] R. M. France, W. E. McMahon, A. G. Norman, J. F. Geisz, and M. J. Romero, "Control of misfit dislocation glide plane distribution during strain relaxation of CuPt-ordered GaInAs and GaInP," J. Appl. Phys., vol. 112, no. 2, p. 023520, 2012.

[3] J. F. Geisz et al., "High-efficiency GaInP/GaAs/nnGaAs triple-junction solar cells grown inverted with a metamorphic bottom junction," Appl. Phys. Lett., vol. 91, no. 2, p. 023502, 2007.

[4] M. A. Green, K. Emery, Y. Hishikawa, W. Warta, and E. D. Dunlop, "Solar cell efficiency tables (version 48)," Prog. Photovolt. Res. Appl., vol. 24, no. 7, pp. 905-913, Jul. 2016.

[5] E. A. Fitzgerald, "Dislocations in strained-layer epitaxy: theory, experiment, and applications," Mater. Sci. Rep., vol. 7, no. 3, pp. 87142, Nov. 1991.

[6] A. G. Cullis, A. J. Pidduck, and M. T. Emeny, "Misfit Dislocation Sources at Surface Ripple Troughs in Continuous Heteroepitaxial Layers,” Phys. Rev. Lett., vol. 75, no. 12, pp. 2368-2371, Sep. 1995.

[7] R. M. France et al., "Reduction of crosshatch roughness and threading dislocation density in metamorphic GaInP buffers and GaInAs solar cells,” J. Appl. Phys., vol. 111, no. 10, p. 103528, 2012.

[8] I. Garcia et al., "Metamorphic III-V Solar Cells: Recent Progress and Potential,” IEEE J. Photovolt., vol. 6, no. 1, pp. 366-373, Jan. 2016. 